\title{
Modeling and prediction of fast CME/shocks associated with type II bursts
}

\author{
H. Xie ${ }^{1,2}$, N. Gopalswamy ${ }^{2}$ and O. C. St. $\mathrm{Cyr}^{2}$ \\ ${ }^{1}$ Catholic University of America, Washington DC, 20064, USA \\ email: hong.xie@nasa.gov \\ ${ }^{2}$ NASA Goddard Space Flight Center, Greenbelt, MD 20771, USA
}

\begin{abstract}
A numerical simulation with ENLIL+Cone model was carried out to study the propagation of the shock driven by the 2005 May 13 CME. We then conducted a statistical analysis on a subset of similar events, where a decameter-hectometric (DH) type II radio burst and a counterpart kilometric type II have been observed to be associated with each CME (DHkm CME). The simulation results show that fast CME-driven shocks experienced a rapid deceleration as they propagated through the corona and then kept a nearly constant speed traveling out into the heliosphere. Two improved methods are proposed to predict the fast CMEdriven shock arrival time, which give the prediction errors of 3.43 and $6.83 \mathrm{hrs}$, respectively.
\end{abstract}

Keywords. Coronal mass ejections (CMEs), shock waves, solar-terrestrial relations.

\section{Introduction}

Many efforts have been made to study the CME and shock propagation using theoretical models (e.g., Chen 1996; Borgazzi et al. 2008), numerical simulations (e.g., Cargill 2004; Odstrcil \& Pizzo 1999; Odstrcil et al. 2005), and empirical models (e.g., Gopalswamy et al. 2001a; Gopalswamy et al. 2005; Cremades et al. 2007; Vrôsnak \& Ẑic 2007). In this work, we use numerical simulations with ENLIL+Cone model (Odstrcil et al. 2005) to conduct a case study of the 2005 May 13 CME. We then study a subset of similar CMEs, where a decameter-hectometric (DH) type II radio burst $(14-1 \mathrm{MHz})$ and a counterpart kilometric $(<1000 \mathrm{kHz})$ type II were observed to be associated with each event (DHkm CME). Two new methods are proposed to improve the prediction of the shock arrival of such CMEs.

\section{Numerical simulation}

The ENLIL+Cone model we use is a three-dimensional MHD model of the heliosphere that forecasts CME/shock propagation from the ENLIL inner boundary $\left(21.5 R_{s}\right)$ to the point of interest. The simulation is done in two stages: 1 ) setup of the steady state ambient solar wind background and 2) insert the transient disturbances (CMEs) propagating in that background. The input CME is specified as a conical plasma cloud with the location, angular width, and velocity corresponding to the cone model fit (Xie et al. 2004) to the coronagraph observations. Recent positive results using this method have been reported by Taktakishvili (2008).

The ENLIL+Cone model has well simulated the kinematic evolution of the 2005 May $13 \mathrm{CME}$ and its driven shock, with a prediction error of the shock arrival of $\sim 4 \mathrm{hrs}$. The CME is a fast halo CME with a M8.0 flare and the CME onset time is 16:47 UT. The observed sky-plane speed and space speed from the CME cone model are $1689 \mathrm{~km} / \mathrm{s}$ and $2171 \mathrm{~km} / \mathrm{s}$, respectively. Figure 1 (left panel) shows that the CME-driven shock 

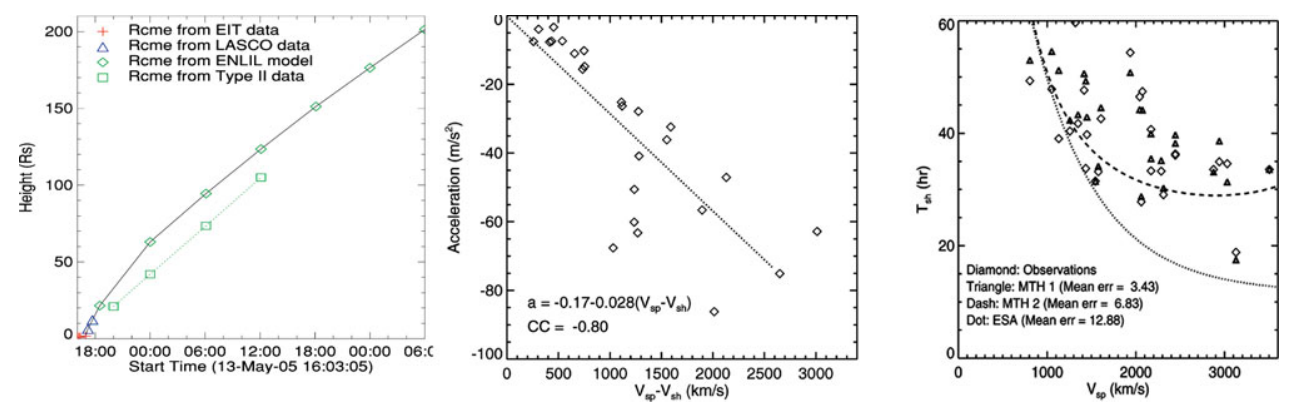

Figure 1. (left) Height-time profile of CME and its driven shock propagation determined from the cone model fit to coronagraph observations and ENLIL simulation.(middle) Shock acceleration vs. $\left(V_{s p}-V_{s h}\right)$ for 25 DHkm CMEs. (right) Comparison of observations and model predictions of $T_{s h}$ vs. $V_{s p}$. Rcme is the CME radial distance, $V_{s p}$ is the shock speed near the Sun, $V_{s h}$ is the shock speed obtained from kmII dynamic spectrum data, $a$ is the shock acceleration, and $\mathrm{CC}$ is the correlation coeffient between $a$ and $\left(V_{s p}-V_{s h}\right)$.

propagates at a nearly constant speed of $\sim 1000 \mathrm{~km} / \mathrm{s}$ after a rapid deceleration within $0.3 \mathrm{AU}$ with an average acceleration of $\sim 50 \mathrm{~m} / \mathrm{s}^{2}$. The linear speed obtained from the simulation is in agreement with the shock speed of $1016 \mathrm{~km} / \mathrm{s}$ derived from kilometric type II data.

\section{Statistical analysis}

\subsection{Data selection}

We study the shock propagation for a subset of CMEs similar to the 2005 May 13 event. The primary criterion we use is that there must be a decameter-hectometric $(\mathrm{DH})$ type II radio burst occurring at frequencies between 14 and $1 \mathrm{MHz}$ and a counterpart kilometric $(\mathrm{km})$ type II occurring at frequencies less than $100 \mathrm{KHz}$ associated with each CME (DHkm CME). DHkm CMEs are faster and wider than the general population of CMEs (e.g., Gopalswamy et al. 2001b). Since there are both DH and km type II bursts associated with each event, we are able to identify a unique CME-shock pair. In addition, we have excluded limb events (solar source within $25^{\circ}$ from limb).

\subsection{Shock propagation}

We assume that shocks driven by fast CMEs decelerate through the outer corona and inner heliosphere until the CMEs reach the kilometric type II (kmII) domain at constant acceleration. The shock acceleration is given as $a=\left(V_{s p}-V_{s h}\right) / t_{1}$, where $V_{s p}$ is the shock speed near the Sun, $V_{s h}$ is the shock speed derived from the frequency draft of kmII, and $t_{1}$ is acceleration cessation time. We approximate the CME space speed obtained from the cone model fit to LASCO observations as the shock speed near the Sun (since the CME leading edges observed in white-light coronagraphs might be in fact compressed shock fronts ahead of CMEs).

Figure 1 (middle panel) shows the scatter plot of shock acceleration vs. $\left(V_{s p}-V_{s h}\right)$ for 25 DHkm CMEs from 2000-2006. The linear fitting gives:

$$
a=-0.17-0.028\left(V_{s p}-V_{s h}\right)
$$

The obtained acceleration magnitudes are consistent with the accelerations from Gopalswamy et al. (2001b), which obtained by a second-order polynomical fitting to 
the height-time plots of a subset of limb CMEs (associated with DH type II bursts) within LASCO C2 and C3 field of view:

$$
a=-30 .+0.0666 u-4.5 \times 10^{-5} u^{2}
$$

where $u$ denotes the CME initial speed.

\subsection{Improved shock prediction methods}

Method 1 uses $V_{s p}$ and $V_{s h}$ to obtain the shock acceleration, a, acceleration cessation time, $t_{1}$, and acceleration cessation distance, $d_{1}$, then computes the shock travel time using (3.1) and the following equations:

$$
\begin{aligned}
& t_{1}=\left(V_{s p}-V_{s h}\right) / a, d_{1}=V_{s p} t_{1}+1 / 2 a t_{1}^{2} \\
& t_{2}=\left(1 A U-d_{1}\right) / V_{s h}, T_{s h}=t_{1}+t_{2}
\end{aligned}
$$

Method 2 uses the formula (3.2) to obtain the CME speed at $t_{1}$ :

$$
V_{s p 1}=V_{s p}+a t_{1}, \quad t_{1}=\frac{-V_{s p}+\sqrt{V_{s p}^{2}+2 a d_{1}}}{a}
$$

where $d_{1}$ is set to be $0.1 \mathrm{AU}\left(21.0 R_{s}\right)$, and then uses $V_{s p 1}$ as input to the Empirical Shock Arrival (ESA) model (Gopalswamy et al. 2005) to compute $T_{s h}$.

Figure 1 (right panel) compares observations and model predictions of $T_{s h}$ vs. $V_{s p}$, where diamonds denote observed $T_{s h}$ vs. $V_{s p}$, triangles denote $T_{s h}$ vs. $V_{s p}$ from method 1 , and dotted and dashed curve represent the prediction from the ESA model and the improved ESA model (method 2), respectively.

\section{Summary}

1) The ENLIL+Cone model can well simulate the kinematic evolution of the 2005 May 13 event. 2) Method 1 combined with kilometric type II data gives a mean prediction error of $3.43 \mathrm{hrs}$, implying that CME-driven shocks travel at near constant speed after a rapid deceleration phase $(<0.5 \mathrm{AU})$. 3) Method 2 improves the ESA model by taking into consideration the fast deceleration phase within the field of view of $\mathrm{C} 3$, decreasing the mean prediction error from 12.88 to $6.83 \mathrm{hrs}$. 4) The ESA model underestimates the deceleration of fast CMEs in the outer corona.

HX was partly supported by NASA (NNX08AD60A).

\section{References}

Borgazzi, A., Lara, A., Romero-Salazar, L., \& Ventura, A. 2008, Geofisica International, 47, 301

Cargill, P. J. 2004, Sol. Phys., 221, 135

Chen, J. 1996, J. Geophys. Res., 101, 27499

Cremades, H., St. Cyr, O. C., \& Kaise, M. L. 2007, Space Weather, 5, S08001

Gopalswamy et al. 2001a, J. Geophys. Res., 106, 29207

Gopalswamy et al. 2001b, J. Geophys. Res., 106, 29219

Gopalswamy et al. 2005, Adv. Space Res., 36, 2289

Odstrcil, D. \& Pizzo, V. J. 1999, J. Geophys. Res., 104, 483

Odstrcil, D., Pizzo, V. J., \& Arge, C. N. 2005, J. Geophys. Res., 110, A02106

Taktakishvili et al. 2008, submitted to Space Weather

Vrŝnak, B. \& Ẑंic T. 2007, A\&A, 423, 717

Xie, H., Ofman, L., \& Lawrence, G. 2004, J. Geophys. Res., 109, A08103 